\title{
Dialogicidad y narratividad en profesores de excelencia y su relación con el aprendizaje
}

\author{
Verónica Alejandra Villarroel Henríquez \\ Universidad del Desarrollo - Concepción - Chile \\ Alejandro Antonio Sánchez Oñate \\ Universidad del Desarrollo - Concepción - Chile
}

\begin{abstract}
Resumen
La presente investigación estudia la dialogicidad y la narratividad del discurso instruccional de profesores de excelencia pedagógica en la Región del Bío-Bío (Chile), con el objetivo de indagar el efecto de estas en el aprendizaje escolar de estudiantes de educación primer a cuarto año de primaria. La muestra incluyó a 459 estudiantes y 15 docentes. Se utilizaron medidas pre y post de rendimiento académico y pruebas de aprendizaje y memoria para los estudiantes, y la observación de clases para las variables del docente; a partir de un Modelo Lineal Jerárquico. Los resultados de este estudio indicaron que las variables individuales explicaban en mayor medida el rendimiento académico logrado por los alumnos, no obstante el discurso instruccional del profesor y la forma de dialogar con sus alumnos tendrían un rol mediador en esta relación. Se discute respecto del poder del lenguaje como instrumento didáctico del docente en la sala de clases.
\end{abstract}

Palabras claves: Comunicación; Aprendizaje; Formación de Profesores.

\section{Dialogicity and narrativity in teachers of excellence and its relation to learning}

\begin{abstract}
This research studies the dialogic and narrative of instructional discourse of teachers teaching excellence in Bío-Bío (Chile), with the aim of investigating the effect of these on the school learning education student's first to fourth year primary. The sample included 459 students and 15 teachers. Pre and post learning and memory tests for students and academic performance measures, and observing classes for teacher's variables were used; from a Hierarchical Linear Model. The results of this study indicated that individual variables explained further academic performance achieved by the students, however the teacher instructional discourse and how to talk with their students would have a mediating role in this relationship. We discuss about the power of language in teachers as a teaching tool in the classroom.
\end{abstract}

Keywords: Communication; Learning; Teaching Work.

\section{Dialogicidade e narratividade em professores de excelência e sua relação}

\section{com a aprendizagem}

\begin{abstract}
Resumo
Na presente pesquisa se estuda a dialogicidade e a narratividade do discurso instrucional de professores de excelência pedagógica na Região do Bío-Bío (Chile), com o objetivo de indagar o efeito destas na aprendizagem escolar de estudantes de educação de primeiro ao quarto ano do ensino básico. A amostra incluiu 459 estudantes e 15 docentes. Utilizaram-se medidas pré e pós rendimento acadêmico e provas de aprendizagem e memória para os estudantes, e a observação de aulas para as variáveis do docente a partir de um Modelo Linear Hierárquico. Os resultados deste estudo indicaram que as variáveis individuais explicavam em maior medida o rendimento acadêmico alcançado pelos alunos, entretanto, o discurso instrucional do professor e a forma de dialogar com seus alunos teriam um papel mediador nesta relação. Discute-se a respeito do poder da linguagem como instrumento didático do docente na sala de aula.
\end{abstract}

Palavras-chave: Comunicação; Aprendizagem; Formação de Professores. 


\section{Introducción}

La naturaleza social del desarrollo psicológico humano ha sido defendida desde distintos autores y puntos de vista (Garton, 1994, Rogoff, 2003, Vygotski, 1979). En este artículo, se pone en relieve el rol las interacciones sociales mediadas por signos en la construcción de la mente social. Se plantea que lo cultural forma parte de la organización de las funciones psicológicas humanas, a nivel intrapersonal e interpersonal, por medio del signo (Santamaría, 1997; Valsiner \& Van der Veer, 2000). No obstante, el significado no depende sólo de la existencia de un signo y su referente, sino de algo más que permite el establecimiento de esta relación. Ese "algo más" es el diálogo social construido con un otro, que sostiene y apoya la relación entre los significantes y sus significados (Pierce, 1894/1998).

La transferencia de esta perspectiva al ámbito educativo involucra concebir el conocimiento como socialmente construido y, en una primera instancia, dependiente de la interacción con otros. Por lo tanto, el estudio del aprendizaje en el ámbito escolar implica comprender las dinámicas de participación y el análisis de las interacciones de profesores y alumnos en la sala de clases. Según Anderson (1997), es a través de esta interacción social que se lleva a cabo un proceso interpretativo, se negocian significados y se comparten distintas perspectivas, logrando así cierto nivel de comprensión mutua.

Para que el proceso de co-construcción del conocimiento e interpretación del contenido se lleve a cabo satisfactoriamente, se requiere que el profesor y los alumnos desarrollen un estado de intersubjetividad, que es lograda cuando algún aspecto de la realidad es atendido conjuntamente por las personas implicadas (Wells, 1999). A partir de este estado de "sintonía recíproca con el otro" se negocian perspectivas y emerge un marco de sentido que es compartido (Rommetveit, 1992).

Considerando que en la educación formal se construyen conocimientos académicos o científicos, el diálogo que se establece entre el profesor y los alumnos requiere de características instruccionales particulares que permitan al profesor hacerse una idea del significado que los alumnos tienen y las asociaciones que han establecido entre conceptos a lo largo de la vida escolar. Para lograr un aprendizaje significativo, coherente y duradero en los alumnos, se necesita que los estudiantes entiendan lo que se les quiere enseñar, conozcan los temas con los que pueden relacionar esa información, y sepan dónde y cómo anclar lo nuevo con lo ya sabido (Ausubel, 1968/1980; Wells, 2004). Desde la tradición constructivista y el enfoque de la psicología cultural, este artículo estudia cómo algunas características del discurso pedagógico que favorecen el aprendizaje escolar.

Los profesores tienen una manera particular de interactuar verbalmente con los alumnos, un estilo propio que forma parte del estilo de enseñanza de cada docente (Undurraga, 2003). Algunos estudios plantean que las diversas características del comportamiento docente son significativamente estables en el tiempo y tienden a ser presentadas de manera similar en los diversos contextos de acción docente (Ausubel, 1968/1980; Cordero, 1990; Prieto, 1998; Undurraga, 2003). En este estudio consideraremos como supuesto que las características del discurso docente tienden a ser estables y presentan relativa independencia del contexto, ya que tienen que ver con un estilo discursivo que se cristaliza en el tiempo, conformando una manera personal de elaborar el conocimiento y la experiencia. De acuerdo a esto, es posible identificar y describir dos dimensiones del discurso docente: a) la dialogicidad instruccional, y b) la narratividad del discurso pedagógico (Tharp \& Gallimore, 1991; Nelson, 1993).

\section{Cualidad dialógica del discurso}

El entendimiento es dialógico por naturaleza, ya que son los procesos interpsicológicos los que están involucrados tanto en la comprensión como en el cambio conceptual (Wertsch, 1991; Vosniadu, 1994). Por estos motivos, las oportunidades que se presentan a los alumnos para hacer preguntas, y comunicar tanto sus pensamientos como sus dudas, son instancias que les permiten compartir significados y al mismo tiempo construir el conocimiento.

En este sentido, Anderson (1997) afirma que la conversación es el vehículo más importante de la construcción de significado. La conversación no es una suerte de objeto fijado de antemano, estable y previsible; sino que cada conversación transcurre de una manera idiosincrática: es desarrollada durante ella y es específica a un contexto, sus participantes y circunstancias. La comprensión es siempre evolutiva y deriva del evento dialógico mismo (Valsiner, 2000).

Tharp y Gallimore (1991) han estudiado la interacción verbal entre profesor y alumnos, distinguiendo entre discusión monológica y conversación instruccional. Para estos autores, la discusión monológica consiste en una serie de preguntas dispersas del profesor que requieren de la respuesta convergente y fáctica del alumno. En cambio, la conversación instruccional es un discurso en el cual el profesor y los estudiantes tejen un habla en conjunto, basada en lecturas previas que permiten crear una oportunidad para el desarrollo conceptual y lingüístico, propiciando el análisis, la reflexión y el pensamiento crítico (Mercer, 2008; Tharp \& Gallimore, 1991; Tharp \& Yamauchi, 1994).

En el polo dialógico instruccional, encontramos docentes que al momento de enseñar interactúan verbalmente con los alumnos, favoreciendo la participación de ellos y la discusión del contenido (Mercer, 2009). En el discurso de estos profesores predomina el diálogo pedagógico, el docente utiliza distintas técnicas que cumplen un papel de andamiaje como, por ejemplo, relacionar la nueva información con lo que los alumnos ya conocen, repetir o parafrasear el discurso de los estudiantes, retomar los comentarios de los alumnos para hacer una síntesis de ellos, promover el relato grupal. Además, comentan y discuten los argumentos de los estudiantes, manteniendo una conversación interactiva con 
ellos, haciendo preguntas abiertas que elicitan la reflexión por parte de los estudiantes.

En cambio, se considerará que el discurso docente está cercano al polo monológico cuando su discurso instruccional es expositivo y no constituye una auténtica conversación con los alumnos. El profesor entrega el contenido de manera estructurada, sin solicitar la participación de los estudiantes ni indagar sobre sus dudas o conceptos previos, determinando de antemano la secuencia temática de la clase, el tiempo y la profundidad con que trata cada tema.

\section{Cualidad narrativa del discurso}

Para Bruner (1998), existen dos modalidades de funcionamiento cognitivo, complementarias pero irreductibles entre sí. Una de las modalidades es la paradigmática (o lógico-científica) que trata de cumplir el ideal de un sistema matemático formal, de descripción y explicación. Su lenguaje está regulado por requisitos de coherencia y no contradicción, mediante axiomas que se analizan de manera deductiva siguiendo un orden lógico. Cada conclusión deriva de otra más general. La modalidad narrativa, en cambio, produce buenos relatos e historias creíbles (aunque no necesariamente "verdaderas"). Se ocupa de las intenciones y acciones humanas, y de las vicisitudes y consecuencias que marcan su transcurso.

La forma en que el profesor enseña tiene peculiaridades que distinguen la labor que hacen los distintos docentes e influye en la cualidad del aprendizaje que obtienen los alumnos. El continuo narrativo-paradigmático del discurso tiene repercusiones en el recuerdo y en la elaboración del conocimiento que llevan a cabo los niños que interactúan con adultos que están más cercanos o lejanos a uno de los polos de esta modalidad. Se esperaría que al interactuar verbalmente con un adulto narrativo, los niños recuerden de mejor forma porque el discurso del adulto logra contextualizar la información que entrega, pero al mismo tiempo y debido a su propiedad subjuntiva, permite que el niño la interprete, signifique y la relacione con su experiencia de vida.

Se considerará que el discurso docente está cercano al polo narrativo del continuo narrativo-paradigmático cuando el profesor muestra en su discurso instruccional aspectos dramáticos, es decir, relata un conjunto de hechos llevados a cabo por ciertos personajes a través de un lenguaje predominantemente descriptivo donde da cuenta de los pensamientos y sentimientos de los protagonistas, identificando la variedad de posibilidades que existen en la trama de la historia y su desenlace. Su narración está organizada temporalmente, presenta relaciones causales a través de episodios que son significativos y contextualizados. En cambio, se considerará que el discurso docente está cercano al polo paradigmático del continuo narrativo-paradigmático cuando el docente muestra un discurso instruccional atemporal y carente de historicidad, presentando una serie de argumentos ordenados lógicamente y fundamentados teóricamente.
Una de las variables fundamentales en el proceso de enseñanza - aprendizaje es la calidad de la interacción que se establece entre profesor y alumno. Bajo el paradigma constructivista sociocultural, la transposición didáctica que establezca el docente será fundamental para el aprendizaje significativo. En particular, el objetivo que persiguió este estudio fue comparar la cualidad del aprendizaje obtenido por los estudiantes cuyos profesores presentan prácticas pedagógicas con alta dialogicidad y/o narratividad en relación a los alumnos que interactúan con docentes con baja dialogicidad y/o narratividad.

\section{Método}

\section{Diseño}

Corresponde a una investigación cuantitativa, en que se utilizó un Modelo Lineal Jerárquico conocido como Hierarchical Linear Modeling (HLM) (Tinsley \& Brown, 2000).

\section{Participantes}

La muestra incluyó a 459 estudiantes, provenientes de 13 colegios de la Región del Bío Bío, que cursaban $1^{\circ}$, $3^{\circ}$ y $4^{\circ}$ básico al momento de ser encuestados ( $46 \%$ en $1^{\circ}$ medio; $32.5 \%$ en $3^{\circ}$ medio y $21.5 \%$ en $4^{\circ}$ básico). El total de profesores considerados fue 15 (1 hombre y 14 mujeres), por lo que la muestra final incluyó 15 cursos. De los profesores incluidos en el estudio, 9 obtuvieron una calificación "competente" y 6 "destacados" en su evaluación de desempeño docente ( 5 en lenguaje y 10 en comprensión del medio social y cultural).

\section{Instrumentos}

Para evaluar la dialogicidad y narratividad de discurso docente, se utilizaron filmaciones de clases de los docentes, las que fueron analizadas mediante dos pautas de observación, una para cada dimensión del discurso instruccional, construidas a partir de los trabajos de Tharp y Gallimore (1991) y Bruner (1998) sobre el discurso dialógico y narrativo, respectivamente.

Respecto a la Dimensión Dialógica Instruccional, se analizaron dos elementos: 1) Instruccionales (ligados a la didáctica favorecer el aprendizaje) y 2) Intersubjetivos (relación profesor-alumno). En los elementos instruccionales se consideran diálogos que el profesor genera con los alumnos que permiten: a) activar conocimientos previos, b) promover lenguaje y expresión oral complejo, c) estimular la argumentación, d) generar nuevas comprensiones y e) elaborar síntesis. En los elementos intersubjetivos se considera el análisis de los diálogos en que el profesor: a) valora e incorpora las contribuciones de los alumnos, b) usa preguntas amplias 
y de respuesta variada y c) hace preguntas individuales y grupales.

Respecto a la Dimensión Narrativa, para determinar cuando un relato era narrativo, se trabajó en primer lugar con la selección del lenguaje conexo del profesor. El lenguaje conexo se refiere al discurso docente, ligado a contenido curricular, y de carácter ininterrumpido. Posteriormente, se determinó la narratividad de cada relato, debiendo cumplir con tres de las siguientes seis características: a) Descripción (incluye la caracterización de las personas involucradas en el relato y el lugar en que ocurre lo que se está contando), b) Subjuntividad (se refiere a la expresión de la realidad de manera i) subjetiva es decir, el relato de los conflictos humanos y el mundo interno de los personajes: lo que saben, piensan, sienten o dejan de pensar y sentir, y ii) subjuntiva, que tiene que ver con el relato de lo que podría ser: lo posible, lo hipotético, lo deseado), c) Acción (se refiere al relato de las acciones que llevan a cabo los personajes, sus metas y motivaciones: el aspecto dramático de la historia), d) Orientación Temporal (se refiere al ordenamiento temporal de los acontecimientos que narra, permitiendo captar el sentido de quién hizo qué a quién, con qué objetivo, con qué resultado, en qué sucesión temporal), e) Causalidad Subjetiva (relación entre los acontecimientos relatados, enfatizando el cómo y porqué de los hechos que narra. No se circunscribe a la causalidad lógica sino a una relación particular entre los hechos). La condición más importante y definitoria de un relato narrativo es la presencia de las características de subjuntividad y acción en un mismo relato.
Dentro del conjunto de variables del estudiante, se evaluó el aprendizaje logrado por los alumnos, utilizando una prueba construida por el equipo de investigadores, basada en contenidos mínimos obligatorios de los cursos con los que se trabajó (primer, tercer y cuarto año básico). La prueba fue sometida a la evaluación de jueces expertos en el tema e investigadores. Los puntajes máximos de la prueba eran: 12 puntos para primer año, 23 puntos para segundo año y 27 puntos para tercer año. Adicionalmente, se evaluó la capacidad de memoria con una prueba construida para el estudio y la comprensión lectora a través de la Prueba de Comprensión Lectora (CLP).

Todos los análisis de datos fueron realizados con los programas estadísticos SPSS 15.0 y HLM 6.02.

\section{Resultados}

En las tablas 1 y 2 se presenta el resumen de la duración de las observaciones realizadas a cada profesor. Todos estos datos están expresados en segundos, y corresponden al promedio de lo observado en dos filmaciones.

La tabla 1 incluye información sobre el nivel del curso $\left(1^{\circ}, 3^{\circ} \circ 4^{\circ}\right.$ año), la dimensión del Discurso Instruccional ( $\mathrm{N}=$ Narratividad, $\mathrm{D}=$ Dialogicidad) y el Elemento Instruccional $(\mathrm{ACP}=$ activa conocimientos previos, $\mathrm{PLC}=$ promueve lenguaje complejo y expresión oral compleja, $\mathrm{EA}=$ estimula argumentación, $\mathrm{GNC}=$ genera nuevas comprensiones y ES= elabora síntesis).

Tabla 1. Resumen duración de observaciones a cada profesor: Curso, discurso instruccional y elemento instruccional.

\begin{tabular}{|c|c|c|c|c|c|c|c|c|}
\hline Profesor & Curso & $\mathbf{N}$ & D & $\mathrm{ACP}$ & PLC & EA & GNC & ES \\
\hline 1 & 1 & 73 & 1255 & 0 & 255 & 613 & 272 & 255 \\
\hline 2 & 4 & 88 & 92 & 0 & 18 & 24 & 56 & 0 \\
\hline 3 & 3 & 0 & 538 & 0 & 103 & 407 & 0 & 29 \\
\hline 4 & 1 & 0 & 286 & 0 & 0 & 225 & 13 & 48 \\
\hline 5 & 3 & 0 & 763 & 0 & 297 & 252 & 110 & 105 \\
\hline 6 & 3 & 0 & 489 & 50 & 150 & 243 & 97 & 0 \\
\hline 7 & 1 & 204 & 1684 & 92 & 866 & 0 & 0 & 0 \\
\hline 8 & 1 & 35 & 91 & 0 & 38 & 0 & 53 & 0 \\
\hline 9 & 1 & 0 & 393 & 0 & 132 & 189 & 0 & 72 \\
\hline 10 & 1 & 11 & 755 & 41 & 254 & 146 & 237 & 78 \\
\hline 11 & 1 & 108 & 466 & 78 & 122 & 177 & 895 & 0 \\
\hline 12 & 3 & 0 & 1158 & 40 & 139 & 238 & 532 & 210 \\
\hline 13 & 4 & 135 & 669 & 41 & 230 & 230 & 179 & 34 \\
\hline 14 & 4 & 0 & 557 & 28 & 38 & 42 & 0 & 58 \\
\hline 15 & 4 & 25 & 1329 & 70 & 663 & 226 & 71 & 300 \\
\hline
\end{tabular}


Tabla 2. Resumen duración de observaciones a cada profesor: Elemento intersubjetivo y función de diálogo.

\begin{tabular}{cccccccc}
\hline Profesor & VCA & UPA & PIG & ARG & REF & NEG & TPO EXP \\
\hline 1 & 270 & 250 & 225 & 677 & 200 & 751 & 3025 \\
2 & 17 & 24 & 51 & 0 & 91 & 0 & 3924 \\
3 & 47 & 0 & 362 & 450 & 41 & 48 & 1582 \\
4 & 153 & 48 & 85 & 24 & 35 & 34 & 1023 \\
5 & 270 & 0 & 475 & 407 & 190 & 274 & 3405 \\
6 & 198 & 21 & 293 & 177 & 211 & 78 & 2243 \\
7 & 284 & 0 & 744 & 285 & 385 & 632 & 4057 \\
8 & 53 & 0 & 38 & 40 & 0 & 160 & 2471 \\
9 & 121 & 69 & 293 & 280 & 0 & 0 & 1347 \\
10 & 143 & 150 & 462 & 343 & 93 & 319 & 3946 \\
11 & 195 & 0 & 271 & 0 & 40 & 454 & 2694 \\
12 & 219 & 403 & 418 & 200 & 247 & 93 & 3574 \\
13 & 511 & 50 & 210 & 241 & 99 & 423 & 3365 \\
14 & 294 & 11 & 124 & 189 & 64 & 141 & 3234 \\
15 & 465 & 34 & 444 & 53 & 152 & 298 & 3790 \\
\hline
\end{tabular}

La tabla 2 agrega información sobre el Elemento Intersubjetivo (VCA = valora e incorpora las contribuciones de los alumnos, UPA= usa preguntas amplias y de respuesta variada y $P I G=$ hace preguntas individuales y grupales) y la Función de Diálogo (ARG= argumentación, REF= reformulación, NEG= negociación). Por último, la columna TPO EXP se refiere al tiempo total de exposición.

Para dar respuesta al objetivo central de esta investigación, referido a indagar si existen diferencias en el aprendizaje de los alumnos a partir del discurso instruccional del profesor, se realizó un análisis descriptivo del puntaje obtenido por los alumnos en el post-test, de acuerdo al estilo dialógico y narrativo del docente.

De los 15 profesores incluidos en el estudio, cuatro de ellos tuvieron un alto nivel de narratividad y un alto nivel de dialogicidad. Estos profesores tuvieron alumnos con promedio de notas de $6,0(S D=0,4)$, puntaje $T$ promedio en el post-test de 49,2 puntos $(S D=5,2)$, puntaje $T$ promedio en memoria de 50,9 puntos $(S D=4,6)$ y un percentil promedio en el test de lenguaje de $43.36(S D=8,2)$.

En cuanto a los profesores con alta dialogicidad y baja narratividad $(n=4)$, es posible señalar que los alumnos de estos docentes tuvieron un promedio de notas de 5,9 ( $S D=$ $0,34)$, puntaje $T$ promedio en el post-test de 47.99 puntos $(S D=2,31)$, puntaje $T$ promedio en memoria de 46,17 puntos $(S D=4,2)$ y un percentil promedio en el test de lenguaje de $44,57(S D=18,02)$.
La categoría de alta narratividad y baja dialogicidad fue obtenida por sólo 1 docente. Los alumnos de este profesor tuvieron un promedio de notas de 5,99 puntos, puntaje $T$ promedio en el post-test de 59,03 puntos, puntaje T promedio en memoria de 55,70 puntos y un percentil promedio en el test de lenguaje de 46,81.

Finalmente, 6 profesores tuvieron un bajo nivel de narratividad y un bajo nivel de dialogicidad. Los alumnos de estos docentes tuvieron un promedio de notas de 5,76 ( $S D=$ $2,9)$, puntaje $T$ promedio en el post-test de 50,56 puntos $(S D=$ $2,92)$, puntaje $T$ promedio en memoria de 51,05 puntos $(S D=$ $1,62)$ y un percentil promedio en el test de lenguaje de 39,30 $(S D=9,08)$. Todos estos resultados se sintetizan en la tabla 3 .

Pese a las diferencias encontradas entre los distintos grupos de profesores, según su grado de narratividad y dialogicidad, la prueba ANOVA indica que las diferencias entre estos grupos respecto a las notas de sus alumnos, el puntaje $T$ en el post-test, el puntaje $T$ en el test de memoria y el percentil en el test del lenguaje de sus alumnos, no resultan estadísticamente significativas.

Debido a la estructura jerárquica de los datos utilizados, es decir, alumnos (características individuales de aprendizaje) anidados en cursos (estilo de enseñanza del docente) se procedió a realizar un segundo tipo de análisis, esta vez de modelamiento jerárquico de los datos (HLM). Este análisis considera cuatro etapas (modelo base, modelo con predictores de Nivel-2; modelo con predictores de $\mathrm{Ni}$ vel-1; y relación entre niveles). 
Tabla 3. Síntesis de resultados de aprendizaje, memoria y lenguaje en estudiantes según discurso instruccional del docente.

\begin{tabular}{|c|c|c|c|c|}
\hline $\begin{array}{l}\text { Discurso Instruccional } \\
\text { Docente }\end{array}$ & $\begin{array}{l}\text { Promedio de notas } \\
\text { de los alumnos }\end{array}$ & $\begin{array}{c}\text { Puntaje } \\
\text { Post Test de } \\
\text { Aprendizaje }\end{array}$ & $\begin{array}{l}\text { Puntaje } \\
\text { Memoria }\end{array}$ & Puntaje CLP \\
\hline $\begin{array}{l}\text { Alto Diálogo / Alta Narración } \\
(\mathrm{N}=4)\end{array}$ & 6.0 & 49.2 & 50.9 & 43.36 \\
\hline $\begin{array}{l}\text { Alto Diálogo / Baja Narración } \\
(\mathrm{N}=4)\end{array}$ & 5.9 & 47.9 & 46.17 & 44.57 \\
\hline $\begin{array}{l}\text { Baja Diálogo / Alta Narración } \\
(\mathrm{N}=1)\end{array}$ & 5.9 & 59.03 & 55.7 & 46.81 \\
\hline $\begin{array}{l}\text { Baja Diálogo / Baja Narración } \\
(\mathrm{N}=6)\end{array}$ & 5.7 & 50.56 & 51.05 & 39.30 \\
\hline
\end{tabular}

Tabla 4. Predictores de nivel individual y colectivo ingresados en primer modelo jerárquico.

\begin{tabular}{ll}
\hline De nivel individual & De nivel colectivo \\
\hline Rendimiento académico del alumno & Tiempo promedio de clase expositiva \\
Porcentaje en prueba CLP & Porcentaje de narratividad \\
Puntaje en prueba de memoria & Porcentaje de dialogicidad \\
Puntaje en pre-test & Porcentaje de diálogo conexo del profesor \\
& Promedio de alumnos del profesor en el SIMCE \\
\hline
\end{tabular}

\section{Primer Modelo Jerárquico}

El primer modelo propuesto consideró como variable dependiente el puntaje obtenido en el post-test de aprendizaje, utilizando los siguientes predictores (Tabla 4):

El promedio estimado para el puntaje $T$ del post-test fue de 50.11 (es=1.074). El 13.58\% $(p=0.00)$ de la variabilidad del puntaje $T$ del post-test corresponde a variabilidad entre los cursos con distintas profesoras; y el $89.42 \%$ a variabilidad individual de los alumnos.

Al ingresar los predictores de segundo nivel, es decir, las variables relacionadas al quehacer de cada docente en su grupo, fue posible apreciar que ninguno de estos factores tiene una relación significativa con el puntaje $T$ obtenido por el alumno en el post-test. Lo anterior, indica que ni el tiempo promedio que el docente realiza clase expositiva, el porcentaje de tiempo de narratividad dentro de la clase expositiva, el porcentaje de tiempo de dialogicidad dentro de la clase expositiva, el porcentaje de tiempo de diálogo conexo dentro de la clase expositiva ni el promedio total en el SIMCE obtenido por los alumnos de su curso están asociados con el aprendizaje final de cada alumno medido en el post-test.

La varianza residual entre grupos con distintos profesores, luego de ingresado los predictores de segundo nivel, es 12.37 , lo que indica que los predictores no contribuyeron a explicar un porcentaje significativo de la variabilidad inicial (13.62661).
Al ingresar los predictores de primer nivel (notas del alumno, puntaje $T$ en test de memoria, puntaje $T$ en el pre-test y puntaje en el test de lenguaje (percentil CLP), es posible apreciar que todas estas variables tienen una asociación estadísticamente significativa y positiva con el puntaje T obtenido por cada alumno en el post-test. Específicamente, respecto a la variable puntaje obtenido en el test de lenguaje (percentil CLP), se tiene que por cada un punto de aumento por sobre el promedio del grupo en el test CLP, el puntaje T en el post-test del alumno aumenta en 0.05 puntos.

En cuanto a la variable "notas", por cada un punto de aumento en la nota del alumno por sobre el promedio del grupo, el puntaje $T$ en el post-test aumenta en 2.46 puntos. Respecto a la variable "puntaje $T$ en memoria", por cada un punto de aumento por sobre el promedio del grupo, el post-test del alumno aumenta en 0.19 puntos. Finalmente, por cada un punto de aumento en el puntaje $T$ obtenido por el alumno en el pre-test (puntaje $T$ ), el puntaje $T$ en el post-test aumenta en 0.28 puntos.

La varianza residual entre individuos, luego de ingresados los predictores, es de 49.66, lo que indica que los predictores explicaron el $41.32 \%$ de la variabilidad inicial (89.74889).

Al ingresar conjuntamente los predictores de primer y segundo nivel, es posible apreciar que el tiempo promedio que el profesor dedica a hacer clase expositiva predice significativamente la pendiente de nivel 1 de la variable "no- 
tas"; mientras que el porcentaje de tiempo que el docente dedica a la dialogicidad dentro de su clase expositiva predice significativamente la pendiente de nivel 1 de la variable "puntaje $T$ en memoria". Lo anterior significa que la relación existente entre el promedio de notas del alumno y su puntaje en el post-test varía de acuerdo a cuánto tiempo promedio el profesor dedica a realizar clase expositiva. Asimismo, la relación existente entre el puntaje $T$ que el alumno obtuvo en el test de memoria y su puntaje en el post-test también varía de acuerdo a cuál es el porcentaje de dialogicidad que presenta el docente dentro de su clase. De este resultado se desprende que el tiempo que el docente dedica a realizar clases expositivas aumentan la fuerza de la relación existente entre el promedio de notas del alumno y el aprendizaje demostrado en el post-test. Esto implica que los alumnos que tienen profesores con tiempos altos en clase expositiva tienen pendientes más altas entre sus notas y el resultado del post-test.

Respecto al porcentaje de dialogicidad del docente dentro de su clase, éste aumentaría la fuerza de la relación existente entre el puntaje $T$ obtenido por el alumno en la prueba de memoria y el aprendizaje demostrado en el post-test. Lo anterior indica que los alumnos cuyos profesores dedican un alto porcentaje de la clase expositiva a la dialogicidad tienen pendientes más altas aún entre su puntaje $T$ en el test de memoria y el resultado del post-test.

\section{Segundo Modelo Jerárquico}

El segundo modelo propuesto consideró, como variable dependiente, el puntaje $T$ obtenido en el post-test de aprendizaje, y consideró los siguientes predictores (Tabla 5):

El promedio estimado para el puntaje $T$ del post-test es $50,84(E S=1,12)$. El 15,99\% $(p<0.01)$ de la variabilidad del puntaje $T$ del post-test corresponde a variabilidad entre los cursos con distintos profesores; y el $84,01 \%$ a variabilidad individual de los alumnos.
Al ingresar los predictores de segundo nivel, es decir, las variables relacionadas al quehacer de cada docente en su curso, es posible apreciar que ninguno de estos factores tiene una relación significativa con el puntaje T obtenido por el alumno en el post-test. Lo anterior, indica que ni la función del diálogo (argumentación, negociación, reformulación), ni el elemento instruccional predominante (activar conocimientos previos, promover el lenguaje complejo y expresión oral compleja, estimular la argumentación, generar nuevas comprensiones, elaborar síntesis), ni el elemento intersubjetivo predominante en el docente (valorar e incorporar las contribuciones de los alumnos, usar preguntas amplias y de respuesta variada, hacer preguntas individuales y grupales) están asociados con el aprendizaje final de cada alumno medido en el post-test.

La varianza residual entre cursos con distintos profesores, luego de ingresar los predictores de segundo nivel, es 3,998 , lo que indica que los predictores en su conjunto contribuyeron a explicar un porcentaje significativo de la variabilidad inicial $(14,36)$; aún cuando individualmente ninguno contribuyó significativamente a la explicación.

Al ingresar los predictores de primer nivel (notas del alumno, puntaje $T$ en test de memoria, puntaje $T$ en el pre-test y puntaje en el test de lenguaje (percentil CLP), es posible apreciar que todas estas variables tienen una asociación estadísticamente significativa y positiva con el puntaje $T$ obtenido por cada alumno en el post-test._Específicamente, respecto a la variable puntaje obtenido en el test de lenguaje, se tiene que por cada un punto de aumento por sobre el promedio del grupo en el test CLP, el puntaje $T$ en el post-test del alumno aumenta en 0.05 puntos.

En cuanto a la variable "notas", por cada un punto de aumento en la nota del alumno por sobre el promedio del grupo, el puntaje $T$ en el post-test aumenta en 2,24 puntos. Respecto a la variable "puntaje $T$ en memoria", por cada un punto de aumento por sobre el promedio del grupo, el post-test del alumno aumenta en 0,19 puntos. Finalmente, por cada un punto de aumento en el puntaje $T$ obtenido por el alumno en el pre-test (puntaje $T$ ), el puntaje $T$ en el post-test aumenta en 0,30 puntos. El promedio estimado para el puntaje $T$ en el

Tabla 5. Predictores de nivel individual y colectivo ingresados en segundo modelo jerárquico.

\begin{tabular}{ll}
\hline De nivel individual & De nivel colectivo \\
\hline Notas del alumno (NOTAS) & Argumentación como función del diálogo \\
Porcentaje en test de lenguaje CLP & Reformulación como función del diálogo \\
Puntaje en prueba de memoria & Negociación como función del diálogo \\
Puntaje en pre-test & Activar conocimientos previos \\
& Promueve lenguaje y expresión oral compleja \\
& Estimula argumentación \\
& Genera nuevas comprensiones \\
& Elabora síntesis \\
& Valora e incorpora las contribuciones de los alumnos \\
& Usa preguntas amplias y de respuesta variada \\
& Hace preguntas individuales y grupales \\
\hline
\end{tabular}


post-test de un alumno con notas promedio, puntaje $T$ en test de memoria promedio, puntaje $T$ pre-test promedio y percentil CLP promedio es de 50,87 ( $E S=1,11$ ). La varianza residual entre individuos, luego de ingresados los predictores, es de 49,63 , lo que indica que los predictores explicaron el $34,25 \%$ de la variabilidad inicial (75.48).

Al ingresar los predictores de primer y segundo nivel, es posible apreciar que el tiempo promedio que el profesor dedica a promover el lenguaje complejo y expresión oral compleja, a estimular la argumentación, a hacer preguntas individuales y grupales y la función de negociación del diálogo predicen significativamente la pendiente de nivel 1 de la variable "notas" de los alumnos; mientras que la función de reformulación del diálogo del docente predice significativamente la pendiente de nivel 1 de la variable "puntaje $T$ en el pre-test) del alumno. Lo anterior significa que la relación existente entre el promedio de notas del alumno y su puntaje en el post-test de aprendizaje varía de acuerdo a cuánto tiempo el profesor dedica a promover el lenguaje y expresión oral compleja, a estimular la argumentación, a hacer preguntas y a negociar en el diálogo. Asimismo, la relación existente entre el puntaje $T$ que el alumno obtuvo en el pre-test y la obtenida en el post-test varía de acuerdo a cuál es el grado de reformulación en el diálogo utilizado por el docente en su clase.

Por otra parte, el tiempo que el profesor dedica a hacer preguntas individuales y grupales y a la negociación en su diálogo aumentan la fuerza de la relación existente entre el promedio de notas del alumno y el aprendizaje demostrado en el post-test. Esto implica que los alumnos que tienen profesores con tiempos altos en estas variables tienden a presentar pendientes más altas entre sus notas y el resultado del post-test que los alumnos con profesores con tiempos bajos en estas variables.

Respecto al tiempo dedicado por el docente a la reformulación, como función del diálogo, éste aumentaría la fuerza de la relación existente entre el puntaje T obtenido por el alumno en el pre-test y el aprendizaje demostrado en el post-test. Lo anterior indica que los alumnos cuyos profesores dedican mucho tiempo de la clase expositiva a la reformulación tienen pendientes más altas aún entre su puntaje $\mathrm{T}$ en el pre-test y el resultado del post-test que los cursos cuyos profesores dedican poco tiempo a la reformulación.

\section{Discusión y conclusiones}

La presente investigación buscó indagar sobre el nivel de aprendizaje logrado por alumnos de enseñanza básica cuyos profesores mostraban un discurso instruccional altamente dialógico y narrativo al momento de enseñar los contenidos curriculares. Se trabajó con una muestra de 15 profesores acreditados en su excelencia pedagógica, en el entendido, que la calidad docente del profesor estaba ya certificada y por lo tanto no sería una variable interviniente en el estudio.

Los resultados de este estudio indicaron que las variables individuales explicaban en mayor medida el ren- dimiento académico logrado por los alumnos en el post test que evaluaba el aprendizaje de los alumnos en la unidad curricular enseñada; no apareciendo dentro de las variables explicativas aquellas que correspondían a las mediciones de los profesores, como son la dialogicidad y narratividad de su discurso.

Si bien este resultado podría ser interpretado como poco alentador, no resulta sorprendente en la medida que, evidentemente, las variables individuales siempre van a pesar de mayor forma en la conducta o desempeño personal. Desde los estudios de Coleman, Campbell, Hobson, McPartland, Mood, Weinfeld, y York (1966) y la investigación en el área de la efectividad escolar, se señalaba que el efecto de características personales de entrada al sistema escolar tenía un impacto central en el logro académico; sin embargo, también es sabido que una de las razones de que esto ocurre es la dificultad de medir las variables ligadas al ámbito escolar. Lo interesante de este estudio es que un $59 \%$ de la variabilidad en el aprendizaje logrado por los alumnos no se explica, al menos, por las variables individuales medidas.

Al realizar nuevos análisis para examinar este porcentaje no explicado por las variables antes mencionadas, aparecen algunos resultados interesantes ligados a la influencia indirecta que tienen algunas características del discurso instruccional docente al interactuar con variables individuales del alumno. En efecto, el discurso instruccional del profesor y la forma de dialogar con sus alumnos tendrían un impacto en el aprendizaje de ellos, a partir de su interacción con las variables individuales medidas. Las variables que destacaron en este tipo de efecto indirecto son: tiempo de la clase expositiva, tiempo destinado a diálogos que construyen conocimiento, tiempo destinados a realizar preguntas individuales y grupales, tiempo destinado a diálogos que promueven la negociación y reformulación de significados ligados al contenido que se enseña.

Resulta de interés subrayar estos datos en la medida que ponen en relieve el rol del profesor en la sala de clases. Es decir, destacan cómo su forma de enseñar, explicar y compartir con los alumnos los contenidos curriculares a través de diálogos activos y centrados en indagar la comprensión que los alumnos van haciendo, momento a momento, sobre el contenido a enseñar, influye en el aprendizaje logrado por los alumnos. Muchas veces, el poder del lenguaje es subestimado como instrumento didáctico, apostando mucho más a los recursos materiales, audiovisuales, tecnológicos o incluso metodológicos si pensamos en trabajo grupal. No obstante, en especial en los primeros años escolares, la enseñanza protagónica del docente parece tener más peso que el rol de los compañeros de clase como instancias de mediación. Por otra parte, en estas variables es posible intervenir y potenciar las prácticas y estilos discursivos docentes que son efectivos para promover aprendizaje. En cambio, es bastante más difícil actuar sobre las variables personales que intervienen el proceso de aprender.

Sin duda, esta investigación tiene oportunidades de mejora respecto a ciertas decisiones metodológicas como la selección de la muestra (ampliar el número de profesores 
que enseñan en un mismo curso), número de filmaciones realizadas por profesor y sistemas de medición de otras variables implicadas en el proceso de aprender. Asimismo, resulta de interés focalizar la mirada en algunos elementos del discurso narrativo y del diálogo a través de la capacitación de profesores en estas áreas, pensando quizás en un diseño experimental de investigación.

No obstante lo anterior, como fortalezas del diseño actual se destaca la utilización de filmaciones y la rigurosidad del análisis cualitativo llevado a cabo para clasificar los diálogos y relatos en las categorías propias del estudio; llegando desde lo cualitativo al dato cuantitativo. Asimismo, el aporte de esta investigación es medir el aprendizaje de los alumnos como variable resultado a partir de su relación con una variable de segundo nivel como es el discurso del profesor. Es decir, intenta evaluar el efecto de una sobre otra, lo no aparece en el común de las investigaciones ligadas al ámbito de la educación.

Respecto al aporte de esta investigación en el ámbito de las políticas públicas de nuestro país, es posible señalar algunas sugerencias en base a una reflexión más profunda del tema. Al analizar las filmaciones de clases de cada profesor, como también sus transcripciones, llama la atención la dificultad de los profesores para dar cuenta del contenido curricular que deben enseñar a sus alumnos, de tal forma que, favorezcan el aprendizaje de ellos. Se tiende a exponer el contenido y repetirlo hasta que los alumnos escriban o lo memoricen, pero no se explica la racionalidad, el significado o la utilidad que existe tras él. Si bien se observa un avance en la incorporación de ciertas estrategias o metodologías de enseñanza (como introducir a clase, preguntar sobre conocimientos previos, realizar trabajos grupales, realizar un cierre de la clase), no se realiza un uso significativo a favor de profundizar la comprensión que los estudiantes logran del contenido a enseñar.

A partir de estudios como este, parece urgente insistir en mejorar la formación inicial del profesorado y de quienes los forman. Hace falta nutrir la formación académica de los profesores tanto de contenido básico como de didáctica (no sólo esto último es relevante sino ambos focos), ya que no se puede esperar aprendizajes significativos en los alumnos si la enseñanza que realiza el docente, lo que hace es repetir mecánica (y no comprensivamente) sólo información. Es vital que el profesor sea una persona altamente competente en el uso del lenguaje oral y escrito, utilizando un vocabulario enriquecido y denotando fluidez en el uso de la lengua.

La acreditación obligatoria de las facultades de educación es un gran paso para lograrlo, sin embargo, no es suficiente. Es necesario contar con mayores mediciones de entrada al ingreso de la educación superior, como también con mecanismos de compensación y refuerzo de ellas cuando se observa que los alumnos ingresan con debilidades en ciertas áreas, en particular en lenguaje y comunicación.

\section{Referencias}

Anderson, H. (1997). Conversation, language and possibilities: a postmodern approach to therapy. New York: Basic Books.

Ausubel, D.P. (1980). Psicología educativa. Un punto de vista cognoscitivo. Méjico: Trillas. (Trabalho original publicado en 1968).

Bruner, J. (1998). Actos de Significado: Más allá de la revolución cognitiva. Madrid: Alianza Editorial.

Coleman, J.S., Campbell, E., Hobson, C., McPartland, J., Mood, A., Weinfeld, R. \& York, R. (1966). Equality of educational opportunity. Washington D.C.: Government Printing Office.

Cordero, M.L. (1990). Los estilos de enseñanza y las dimensiones de la acción didáctica. Em C. Coll, J. Palacios, \& A. Marchesi (Orgs.), Desarrollo psicológico y educación II. Psicología de la educación (273-295). Madrid: Alianza.

Garton, A.F. (1994). Interacción social y desarrollo del lenguaje y la cognición. Buenos Aires: Educación Paidós.

Nelson, K. (1993). The psychological and social origins of autobiographical memory. Psychological Science, 4 (1), 7-15.

Mercer, N. (2008). Classroom dialogue and the teacher's role. Education Review, 21 (1), 60-65.

Mercer, N. (2009). The analysis of classroom talk: methods and methodologies. British Journal of Educational Psychology, 80, $1-14$

Pierce, C.S. (1894/1998). What Is a Sign? In N. Houser \& C. J. W. Kloesel (Ed.), The Essential Pierce. Bloomington, IN: Indiana University Press.

Prieto, M.D. (1998). Variables del profesor como mediador del proceso instruccional. Em J. Beltrán \& C. Genovard (Orgs.), Psicología de la Instrucción. I Variables y procesos básicos (194-216). Madrid: Síntesis.

Rogoff, B. (2003). The cultural nature of human development. New York: Oxford University Press.

Rommetveit, R. (1992). Outlines of dialogically based social-cognitive approach to human cognition and communication. Em A.H. Wold (Org.), The Dialogical Alternative: Towards a theory of language and mind (19-43). Stockholm: Scandinavian University Press.

Santamaría, A. (1997). La naturaleza semiótica de la conciencia: una aproximación sociocultural a la mente humana, Infancia y Aprendizaje, 80, 3-15.

Tharp, R. \& Gallimore, R. (1991). The instructional conversation: teaching and learning in social activity. Center for Research on Education, Diversity \& Excellence. UC Berkeley: Center for 
Research on Education, Diversity and Excellence. Recuperado: 1 jun. 2016. Disponível: http://files.eric.ed.gov/fulltext/ED341254.pdf

Tharp, R. \& Yamauchi, L. (1994) Effective Instructional Conversation in Native American Classrooms. National Center for Research on Education, Diversity and Excellence. Educational Practice. Recuperado: 1 jun. 2016. Disponível: http://files.eric.ed.gov/ fulltext/ED372896.pdf

Tinsley, E. A. \& Brown, S. (2000). Handbook of applied multivariate statistics and mathematical modeling. San Diego: Academic Press.

Undurraga, C. (2003). ¿Cómo aprenden los adultos? Una mirada psicoeducativa. Santiago de Chile: Ediciones de la Pontificia Universidad Católica de Chile.

Valsiner, J. (2000). Culture and Human Development. London: SAGE Publications.
Valsiner, J. \& Van der Veer, R. (2000). The social mind. Construction of the idea. Cambridge: University Press.

Vosniadu, S. (1994). Capturing and modelling the process of conceptual change. Learning and Instruction, 4, 45-69.

Vygotski, L.S. (1979). El desarrollo de los procesos psicológicos superiores. Barcelona: Editorial Crítica.

Wells, G. (1999). Language and Education: reconceptualizing education as dialogue. Annual Review of Applied Linguistics, 19 135-155.

Wells, G. (2004). El papel de la actividad en el desarrollo y la educación. Infancia y Aprendizaje, 27 (2), 165-187.

Wertsch, J. (1991). Voices of the mind. A sociocultural approach to mediated action. Massachusetts: Harvard University Press.

Recebido em: 10 de junho de 2016

Aprovado em: 16 de maio de 2017

\section{Sobre os autores}

Verónica Alejandra Villarroel Henríquez (vvillarroel@udd.cl)

Psicóloga, Magíster en Psicología Educacional, Doctora (PhD) en Psicología. Universidad del Desarrollo - Concepción-Chile.

Alejandro Antonio Sánchez Oñate (alesanchezo@udd.cl)

Psicólogo, Magíster (c) en Investigación Social y Desarrollo. Universidad del Desarrollo - Concepción-Chile.

Esta investigación recibió el apoyo financiero del Ministerio de Educación de Chile, a través del $2^{\circ}$ Concurso del Fondo de Investigación y Desarrollo en Educación - FONIDE. 